PROCEEDINGS OF THE

AMERICAN MATHEMATICAL SOCIETY

Volume 126, Number 7, July 1998, Pages 2117-2122

S 0002-9939(98)04307-X

\title{
RELATIVE TO ANY NONRECURSIVE SET
}

\author{
THEODORE A. SLAMAN
}

(Communicated by Andreas R. Blass)

\begin{abstract}
There is a countable first order structure $\mathfrak{M}$ such that for any set of integers $X, X$ is not recursive if and only if there is a presentation of $\mathfrak{M}$ which is recursive in $X$.
\end{abstract}

\section{INTRODUCTION}

It is a well-known truth that if a set $R \subseteq \omega$ is recursive relative to every nonrecursive subset of $\omega$, then $R$ is recursive (see [Kleene and Post, 1954] ). Similarly, if $R$ is recursive in every element of a co-meager set, then $R$ is recursive; if $R$ is recursive in every element of a set of positive measure, then $R$ is recursive; and if $R$ is recursive in every element of a nonempty $\Pi_{1}^{0}$ set $P$, then $R$ is recursive in the reals appearing in the definition of $P$. In fact, one can consider a wide variety of notions of forcing $\mathbb{P}$, and conclude that if $R$ is recursive in every generic set (extending the condition $p$ ), then $R$ is recursive in $\mathbb{P}$ (and $p$ ). Thus, in wide generality, there is no $R \subseteq \omega$ which constitutes information common to all nonrecursive reals or to all generic reals.

Even so, one need not abandon the possibility that there is a mathematical object which instantiates the property of being nonrecursive. The following question of S. Lempp suggests one such possibility.

Question 1.1 (Lempp). Does there exist a countable first order structure $\mathfrak{M}$ such that, for every $X, X$ is nonrecursive if and only if there is a presentation of $\mathfrak{M}$ which is recursive in $X$ ?

In Section 2, we answer Question 1.1 by showing that there is such an $\mathfrak{M}$. In Theorem 2.1 , we construct an $\mathfrak{M}$ which has no recursive presentation and is uniformlyrecursively presented relative to every nonrecursive real.

Independently and shortly before our obtaining Theorem 2.1, Wehner [Wehner, 1996] answered a question of J. Knight, and obtained an example of a similar type. In the following, say that a family of sets $\mathcal{F}$ is uniformly-recursively enumerable if there is a recursively enumerable set of pairs $W$ such that

$$
(\forall F)[F \in \mathcal{F} \Longleftrightarrow(\exists i)[F=\{n:(i, n) \in W\}]] .
$$

Received by the editors May 10, 1996 and, in revised form, December 17, 1996.

1991 Mathematics Subject Classification. Primary 03C57, 04D45.

Key words and phrases. Recursive model theory.

During the preparation of this paper, Slaman was partially supported by National Science Foundation Grant DMS-9500878. 
Theorem 1.2 (Wehner). There is a family $\mathcal{F}$ of subsets of $\omega$ which is not uniformlyrecursively enumerable, such that for all $X, X$ is nonrecursive if and only if there is a uniformly-recursive-in- $X$ enumeration of $\mathcal{F}$.

Shortly after our obtaining Theorem 2.1, A. McAllister showed that Theorem 2.1 can be derived from Wehner's theorem. The $\Sigma_{1}$-types of the elements of McAllister's model correspond to the sets in Wehner's family. Conversely, it is not difficult to deduce Wehner's theorem by analyzing the $\Sigma_{1}$-types realized in our model.

\section{THE MODEL}

Here, we present the central result of this paper.

Theorem 2.1. There is a countable model $\mathfrak{M}$ and there is a recursive function $M: X \mapsto M(X)$, which maps subsets of $\omega$ to presentations of countable first order structures with the following properties.

(1) For all $X$, if $X$ is not recursive, then $M(X)$ is a presentation of $\mathfrak{M}$.

(2) There is no recursive presentation of $\mathfrak{M}$.

In this section, we will specify the language of $\mathfrak{M}$ and describe the general features of $\mathfrak{M}$, construct $\mathfrak{M}$, and then prove that $\mathfrak{M}$ has the required properties.

2.1. Describing $\mathfrak{M}$. The language of $\mathfrak{M}$ consists of a single constant symbol 0 , a unary function symbol $s$, and three binary relation symbols $R,<_{T}$ and $<_{L}$. We will write $R(x, y), x<_{T} y$, and $x<_{L} y$. Let $\mathcal{L}$ denote this language.

In $\mathfrak{M}$, we use 0 and $s$ to represent the natural numbers with successor. That is, we let $s^{i}(0)^{\mathfrak{M}}$ denote the $i$ th iterate of $s^{\mathfrak{M}}$ applied to $0^{\mathfrak{M}}$, and set $s^{\mathfrak{M}}$ to be the identity on all points not in $\left\{s^{i}(0): i \in \omega\right\}^{\mathfrak{M}}$. We use $<_{T}^{\mathfrak{M}}$ to represent a countable family of nonempty binary trees, and informally let $T_{i}^{\mathfrak{M}}$ denote the $i$ th tree $\left\{x: x>_{T} s^{i}(0)\right\}^{\mathfrak{M}}$ with the ordering given by $<_{T}^{\mathfrak{M}}$. We let $<_{L}^{\mathfrak{M}}$ uniformly order the levels of the $T_{i}^{\mathfrak{M}}$ 's.

Finally, we use $R^{\mathfrak{M}}$ to pick out paths within $T^{\mathfrak{M}}$, as follows . Let $R^{-1}\left(T_{i}\right)^{\mathfrak{M}}$ denote $\left\{p: R\left(p, s^{i}(0)\right)\right\}^{\mathfrak{M}}$. For each $p$ in $R^{-1}\left(T_{i}\right)^{\mathfrak{M}}$, we let $\zeta(p)^{\mathfrak{M}}$ denote the set $\{x: R(p, x)\}^{\mathfrak{M}}$. We will ensure that $\zeta(p)^{\mathfrak{M}}$ is a maximal finite path in $T_{i}^{\mathfrak{M}}$. Further, we will ensure that there are maximal finite paths in $T_{i}^{\mathfrak{M}}$, and ensure that for each maximal finite path $\zeta$ in $T_{i}^{\mathfrak{M}}$ there are infinitely many $p$ such that $\zeta=\zeta(p)^{\mathfrak{M}}$ and so $R^{-1}\left(T_{i}\right)^{\mathfrak{M}}$ is infinite.

In Figure 1, we show a finite subset of the diagram of $\mathfrak{M}$. In this picture, $\zeta(p)^{\mathfrak{M}}$ is equal to the collection of points below $x$ in the sense of $<_{T}^{\mathfrak{M}}$.

Let $\left\langle\mathfrak{R}_{i}, i \in \omega\right\rangle$ be a recursive enumeration of the recursively-enumerably presented $\mathcal{L}$-structures.

We will ensure that either $R^{-1}\left(T_{i}\right)^{\Re_{i}}=\emptyset$, or $\left\langle T_{i},<_{L} \mid T_{i}\right\rangle^{\Re_{i}}$ is not isomorphic to $\left\langle T_{i},<_{L}\left\lceil T_{i}\right\rangle^{\mathfrak{M}}\right.$, or there is a $p$ in $R^{-1}\left(T_{i}\right)^{\mathfrak{R}_{i}}$ such that $\zeta(p)^{\mathfrak{R}_{i}}$ is not maximal, or there is a $p$ in $R^{-1}\left(T_{i}\right)^{\mathfrak{R}_{i}}$ such that $\zeta(p)^{\mathfrak{R}_{i}}$ is infinite. Since none of these disjuncts apply to $\mathfrak{M}$, we will thus ensure that $\mathfrak{M}$ has no recursive presentation.

Although we have been discussing $\mathfrak{M}$, we will only define $\mathfrak{M}$ at the end of our proof. During the proof, we will define a $\Sigma_{1}^{0}$ function $M$, mapping subsets $X$ of $\omega$ to relatively recursively enumerable presentations of models $M(X)$, interpreting the language $\mathcal{L}$. Since a recursively enumerable presentation of an infinite structure can be uniformly converted to a recursive presentation of the same structure, we can conclude that there is a uniformly recursive function mapping $X$ to a recursive presentation of $M(X)$. 


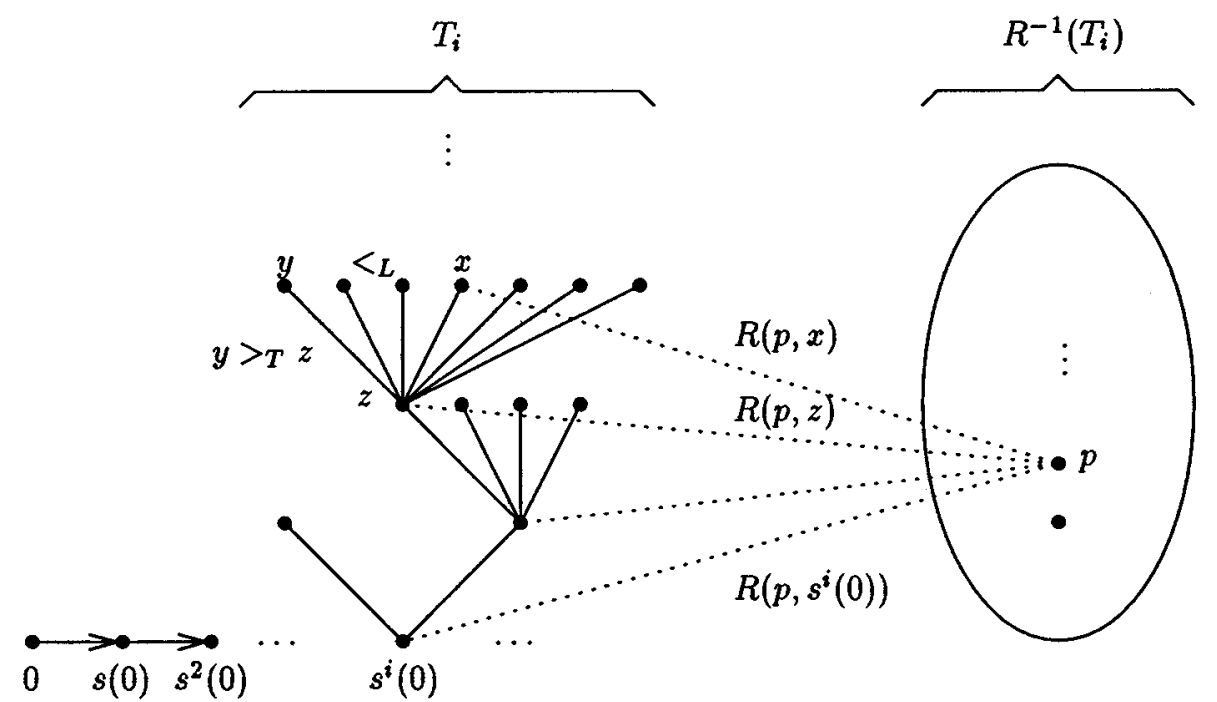

Figure 1. Part of the diagram of $\mathfrak{M}$

Our intention is to ensure that if $X$ is not recursive, then for all $i$ and all $p$ in $R^{-1}\left(s^{i}(0)\right)^{M(X)}, \zeta(p)^{M(X)}$ is a maximal finite path in $T_{i}^{M(X)}$. To diagonalize against all of the recursive isomorphism types, we may make $T_{i}^{M(X)}$ infinite, and so $T_{i}^{M(X)}$ may have an infinite path as well. For arbitrary $X$, we cannot avoid the possibility that $\zeta(p)^{M(X)}$ may be infinite, so we ensure that if $\zeta(p)^{M(X)}$ is infinite, then $X$ is recursive. To this end, we ensure that $T_{i}^{\mathfrak{M}}$ is recursively isomorphic to a recursive tree with at most one infinite path, which would necessarily be recursive. Then, we make the function $M$ sufficiently injective so that for each $p \in \omega$, there can be at most one $X$ such that $\zeta(p)^{M(X)}$ is an infinite path in $T_{i}^{M(X)}$. Then, this $X$ is recursive.

2.2. Constructing $\mathfrak{M}$. We divide our construction of $M(X)$ into two parts, the part that is recursively enumerable without reference to $X$ and and the part that is recursively enumerable with essential references to $X$.

2.2.1. The part of $M(X)$ which does not refer to $X$. We begin by recursively enumerating the diagram of a model $M$, which will interpret $0, s,<_{T}$, and $<_{L}$. Only the interpretation of $R$ within $M(X)$ will depend on $X$.

We let 0 and $s$ be a recursive presentation of the natural numbers with 0 and the successor function such that the domain of $s$ is a recursive coinfinite set.

We enumerate $<_{T}$ above $s^{i}(0)$ while monitoring the $i$ th recursive model $\mathfrak{R}_{i}$ of the same signature as $\mathfrak{M}$. In the following, we use the suffix "[s]" to denote a finite approximation given by what has been enumerated by stage $s$. For example, $\mathfrak{R}_{i}[s]$ is the finite subset of the diagram of $\Re_{i}$ enumerated by stage $s$, and $T_{i}^{M}[s]$ is the finite subtree of $T_{i}^{M}$ which we have enumerated by stage $s$. Now, we enumerate the remaining part of $M$ as follows.

1. (a) While there is a no $x$ within $\mathfrak{R}_{i}[s]$ such that $\mathfrak{R}_{i}[s] \models R\left(x, s^{i}(0)\right)$, we do not enumerate any successors of $s^{i}(0)$. 
(b) If there is an element $x$ within $\mathfrak{R}_{i}[s]$ such that $\mathfrak{R}_{i}[s] \models R\left(x, s^{i}(0)\right)$, then let $p_{0}$ be the first such in the enumeration of $\Re_{i}$.

2. (a) While either the reduced structure $\left\langle T_{i},<_{L}\left\lceil T_{i}\right\rangle^{\Re_{i}}[s]\right.$ is not isomorphic to $\left\langle T_{i},<_{L} \backslash T_{i}\right\rangle^{M}[s]$ or the set $\zeta\left(p_{0}\right)^{\mathfrak{R}_{i}}[s]$ is not a maximal chain in $T_{i}^{\Re_{i}}[s]$, we do not enumerate any further elements into $T_{i}^{M}$.

(b) Otherwise, we let $\pi$ denote the unique isomorphism mapping $\left\langle T_{i}\right.$, $<_{L}\left\lceil T_{i}\right\rangle^{M}[s]$ to $\left\langle T_{i},<_{L} \backslash T_{i}\right\rangle^{\Re_{i}}[s]$. We let $z_{i}^{M}[s]$ be the maximal element of $T_{i}^{M}[s]$ such that $\pi\left(z_{i}^{M}[s]\right)$ is the maximal element of $\zeta\left(p_{0}\right)^{\Re_{i}}[s]$. We enumerate $2^{s}$ many immediate $<_{T}$-successors of $z_{i}^{M}[s]$ into $T_{i}$ and extend $<_{L}^{M}$ so that it linearly orders these new elements of $T_{i}^{M}$. At the next stage, we return to $2(\mathrm{a})$.

The effect of our enumeration is that either the structures $\left\langle T_{i},<_{L}\left\lceil T_{i}\right\rangle^{\Re_{i}}\right.$ and $\left\langle T_{i},<_{L} \uparrow T_{i}\right\rangle^{M}$ are not isomorphic, or they are isomorphic by a recursive isomorphism $\pi:\left\langle T_{i},<_{L}\left\lceil T_{i}\right\rangle^{M} \rightarrow\left\langle T_{i},<_{L}\left\lceil T_{i}\right\rangle^{\mathfrak{R}_{i}}, T_{i}^{M}\right.\right.$ is an infinite finitely-branching tree, and $\pi^{-1}\left(\zeta\left(p_{0}^{\Re_{i}}\right)\right)$ is isomorphic to its unique branch.

Note, we may safely assume that our enumeration of $M$ proceeds so that the universe of $M$ is a co-infinite recursive subset of $\omega$.

2.2.2. The part of $M(X)$ which refers to $X$. We begin by fixing some notation. Let $\preceq$ be the lexicographic ordering of $2^{<\omega}$, and for $\sigma$ in $2^{s}$ let $|\sigma| \preceq$ be the number of elements of $2^{s}$ which are less than or equal to $\sigma$ under $\preceq$.

We define $R^{M(X)}$ by recursively enumerating a collection of pairs $\langle\sigma,\langle p, z\rangle\rangle$ into $W_{R}$, where $\sigma$ is a finite binary sequence, meant to be an initial segment of $X$, and $\langle p, z\rangle$ is a pair, meant to belong to $R^{M(X)}$. Thus, $\langle p, z\rangle$ is an element of $R^{M(X)}$ if and only if there is a finite initial segment $\sigma$ of $X$ such that $\langle\sigma,\langle p, z\rangle\rangle$ is an element of $W_{R}$. During stage $s$, we proceed as follows.

1. (a) While there is a no $x$ within $\mathfrak{R}_{i}[s]$ such that $\mathfrak{R}_{i}[s] \models R\left(x, s^{i}(0)\right)$, we execute only Step 3, below.

(b) If there is an element $x$ within $\mathfrak{R}_{i}[s]$ such that $\mathfrak{R}_{i}[s] \models R\left(x, s^{i}(0)\right)$, then let $p_{0}$ be the first such in the enumeration of $\Re_{i}$, and proceed to Step 2 .

2. (a) While either the reduced structure $\left\langle T_{i},<_{L}\left\lceil T_{i}\right\rangle^{\Re_{i}}[s]\right.$ is not isomorphic to $\left\langle T_{i},<_{L} \uparrow T_{i}\right\rangle^{M}[s]$ or $\zeta\left(p_{0}\right)^{\Re_{i}}[s]$ is not a maximal chain in $T_{i}^{\Re_{i}}[s]$, we execute only Step 3.

(b) Otherwise, we let $\pi$ denote the unique isomorphism mapping $\left\langle T_{i}\right.$, $<_{L}\left\lceil T_{i}\right\rangle^{M}[s]$ to $\left\langle T_{i},<_{L} \mid T_{i}\right\rangle^{\Re_{i}}[s]$. We let $z_{i}^{M}[s]$ be the maximal element of $T_{i}^{M}[s]$ such that $\pi\left(z_{i}^{M}[s]\right)$ is the maximal element of $\zeta\left(p_{0}\right)^{\mathfrak{R}_{i}}[s]$. For each $\sigma$ in $2^{s}$, let $k_{\sigma}$ be the $|\sigma|_{\preceq}$ th immediate successor of $z_{i}^{M}[s]$ (added during stage $s$, as above); for each $p$ such that $R^{\sigma}\left(p, z_{i}^{M}[s]\right)$ holds during stage $s$, enumerate the pair $\left\langle\sigma,\left\langle p, k_{\sigma}\right\rangle\right\rangle$ into $W_{R}$.

3. For each maximal chain $\zeta$ in $T_{i}^{M}[s]$ and for each $\sigma$ in $2^{s}$, we choose a number $p^{*}$ greater than any number which has previously been mentioned in the construction, and for each point $z$ in $\zeta$ enumerate $\langle\sigma,\langle p, z\rangle\rangle$ into $W_{R}$.

Definition 2.2. A countable extension $M^{*}$ of $M$ to an $\mathcal{L}$-structure is appropriate if the following conditions hold.

1. $0, s,<_{T}$ and $<_{L}$ have identical interpretations in $M$ and $M^{*}$.

2. For each $p$ and $z$ in $M^{*}$, if $M^{*} \models R(p, z)$, then $p \in M^{*} \backslash M$.

3. For each $p$ in $M^{*} \backslash M,\left\{z: M^{*} \models R(p, z)\right\}$ is a maximal path in one of the $T_{i}^{M}$. 

$\mathfrak{M}$.

By virtue of our construction, for every $X, M(X)$ is an appropriate extension of

\subsection{Verifying the theorem.}

Lemma 2.3. Suppose that $\mathfrak{M}_{1}$ and $\mathfrak{M}_{2}$ are two appropriate extensions of $M$ such that for $k$ equal to 1 or 2 ,

1. for every $i$ and every maximal finite path $\zeta$ in $T_{i}$, there are infinitely many $p$ in $\mathfrak{M}_{k}$ such that $\zeta(p)^{\mathfrak{M}_{k}}=\zeta$;

2. and there is no $p$ in $\mathfrak{M}_{k}$ such that $\zeta(p)^{\mathfrak{M}_{k}}$ is infinite.

Then, $\mathfrak{M}_{1}$ and $\mathfrak{M}_{2}$ are isomorphic.

Proof. Each of $\mathfrak{M}_{1}$ and $\mathfrak{M}_{2}$ consists of $M$ and infinitely many additional elements which it relates to the elements in $M$ by its interpretation of $R$. Any bijection between the new elements of $\mathfrak{M}_{1}$ and the new elements of $\mathfrak{M}_{2}$ which preserves $R$ will then be an isomorphism.

Each new element $p$ of $\mathfrak{M}_{1}$ is uniquely associated with a maximal finite path $\zeta=\left\{z \in T_{i}: R(p, z)\right\}$ contained in some $T_{i}$. Further, for each such $\zeta$ there are infinitely many $p$ such that $\zeta=\left\{z \in T_{i}: R^{\mathfrak{M}_{1}}(p, z)\right\}$. The same conditions hold for $\mathfrak{M}_{2}$.

Let $\pi$ be any bijection between $\mathfrak{M}_{1} \backslash M$ and $\mathfrak{M}_{2} \backslash M$ so that for each $p$ in $\mathfrak{M}_{1} \backslash M$,

$$
\zeta(p)^{\mathfrak{M}_{1}}=\zeta(\pi(p))^{\mathfrak{M}_{2}} .
$$

Since the only pairs in $R$ are those which associate new elements outside of $M$ to paths in the $T_{i}$, for all $x$ and $y, R^{\mathfrak{M}_{1}}(x, y)$ if and only if $R^{\mathfrak{M}_{2}}(\pi(x), \pi(y))$. Thus, $\mathfrak{M}_{1}$ and $\mathfrak{M}_{2}$ are isomorphic.

Definition 2.4. Let $\mathfrak{M}$ be the isomorphism type of the extension of $M$ to a model of the type mentioned in Lemma 2.3

Corollary 2.5. If for each $p$ in $M(X) \backslash M, \zeta(p)$ is finite, then $M(X)$ is isomorphic to $\mathfrak{M}$.

Proof. Apply Lemma 2.3.

Lemma 2.6. There is no recursive presentation of $\mathfrak{M}$.

Proof. Suppose that $\mathfrak{M}$ has a recursive presentation, and fix $i$ so that $\mathfrak{R}_{i}$ is isomorphic to $\mathfrak{M}$. Since $R^{-1}\left(s^{i}(0)\right)^{\mathfrak{M}}$ is not empty, we may let $p_{0}$ be the first element in the enumeration of $R^{-1}\left(s^{i}(0)\right)^{\Re_{i}}$.

In Section 2.2.1, we defined $T_{i}$ in the following way. We waited for an element to appear in $R^{-1}\left(\left\{s^{i}(0)\right\}\right)^{\Re_{i}}$. Given that $p_{0}$ is the first such element, we then ensured that either $T_{i}^{M}$ is finite and $\zeta\left(p_{0}\right)^{\Re_{i}}$ is not a maximal path in $T_{i}^{\Re_{i}}$, or $\zeta\left(p_{0}\right)^{\Re_{i}}$ is the unique infinite path in $T_{i}^{\Re_{i}}$.

For every element $p$ of $R^{-1}\left(T_{i}\right)^{\mathfrak{M}}, \zeta(p)^{\mathfrak{M}}$ is a finite maximal chain in $T_{i}^{\mathfrak{M}}$. But then, $p$ cannot be isomorphic to $p_{0}$, since $\zeta\left(p_{0}\right)^{\mathfrak{R}_{i}}$ is either not maximal or is infinite.

Lemma 2.7. For each $X$ and $i \in \omega$, one of the following conditions holds.

1. For every $p \in R^{-1}\left(T_{i}\right)^{M(X)}, \zeta(p)^{M(X)}$ is a finite maximal path in $T_{i}^{M}$.

2. $X$ is recursive. 
Proof. As mentioned above, we defined $T_{i}^{M}$ in the following way. We waited for an element to appear in $R^{-1}\left(\left\{s^{i}(0)\right\}\right)^{\mathfrak{R}_{i}}$. For the first such element, $p_{0}$, to appear in the enumeration of $\mathfrak{R}_{i}$, we ensured that either $T_{i}^{M}$ is finite and either $T_{i}^{\mathfrak{R}_{i}}$ is not isomorphic to $T_{i}^{M}$ or $\zeta\left(p_{0}\right)^{\mathfrak{R}_{i}}$ is not a maximal path in $T_{i}^{\mathfrak{R}_{i}}$, or $T_{i}^{M}$ is infinite and $\zeta\left(p_{0}\right)^{\mathfrak{R}_{i}}$ is the unique infinite path in $T_{i}^{\Re_{i}}$.

Item (1) is a clear consequence of the first case. So we may assume that the second case occurs.

In Section 2.2.2, we defined $R$ as follows. If during stage $s, \zeta\left(p_{0}\right)^{\mathfrak{R}_{i}}[s]$ increases in length to become an maximal chain in $T_{i}^{\Re_{i}}[s]$ and $\pi$ is the isomorphism from $T_{i}^{M}[s]$ to $T_{i}^{\Re_{i}}[s]$, then for each $\sigma$ in $2^{s}$ and each $p$ such that $\pi\left(\zeta(p)^{M(\sigma)}\right)[s]$ is equal to $\zeta\left(p_{0}\right)^{\Re_{i}}[s]$, we ensure that for each $X$ extending $\sigma$, only one of the immediate predecessors of the maximum of $\zeta(p)^{M(\sigma)}[s]$ is related to $p$ by $R^{M(X)}$, namely $k_{\sigma}$. Note that we choose $k_{\sigma}$ as an injective function of $\sigma$.

Now, suppose that $X$ and $p$ are given so that $\pi\left(\zeta(p)^{M(X)}\right)=\zeta\left(p_{0}\right)^{\mathfrak{R}_{i}}$. We can compute $X$ as follows. Given a number $x$, wait for a stage $s$ in the construction such that $s>x$ and $\zeta\left(p_{0}\right)^{\Re_{i}}[s]$ is larger than ever before. During stage $s$, we enumerated $R$ so that for each extension $\zeta$ of $\pi^{-1}\left(\zeta\left(p_{0}\right)^{\Re_{i}}\right)[s]$ in $T_{i}^{M}$, there is at most one binary sequence $\sigma$ of length $s$ such that $R(\sigma,\langle p, k\rangle)$ holds for all of the elements $k$ appearing in $\zeta$. But then, there is only one such $\zeta$ such that $\pi(\zeta)$ is an initial segment of $\zeta\left(p_{0}\right)^{\Re_{i}}[s]$, and only one such $\sigma$ for which $R(\sigma,\langle p, k\rangle)$ holds for all of the elements $k$ appearing in that $\zeta$. Since $\zeta(p)^{M(X)}$ is infinite, this unique $\sigma$ must be an initial segment of $X$, and $X(x)$ is equal to $\sigma(x)$.

We can now prove Theorem 2.1. If $X$ is not recursive, then Lemma 2.7 states that there is no $p$ such that $\zeta(p)^{M(X)}$ is infinite. But then Corollary 2.5 states that $M(X)$ is isomorphic to $\mathfrak{M}$. Thus, we can conclude the first claim of Theorem 2.1, that we have a uniformly recursive method to present $\mathfrak{M}$ relative to any nonrecursive set $X$. By Lemma 2.6, there is no recursive presentation of $\mathfrak{M}$, and we can conclude the remaining claim of Theorem 2.1.

\section{REFERENCES}

[Kleene and Post] Kleene, S. C. and Post, E. L. [1954]. The upper semi-lattice of degrees of recursive unsolvability, Anal. Math. 59: 379-407. MR 15:772a

[Wehner] Wehner, S. [1996]. Enumerations, countable structures and Turing degrees, Proc. Amer. Math. Soc. 126 (1998), 2131-2139. CMP 97:11

Department of Mathematics, The University of Chicago, Chicago, Illinois 60637

E-mail address: ted@math.uchicago.edu

Current address: Department of Mathematics, University of California, Berkeley, California 94720-3840 